\title{
BMJ
}

\section{Prevalence of xenotropic murine leukaemia virus-related virus in patients with chronic fatigue syndrome in the Netherlands: retrospective analysis of samples from an established cohort}

\begin{abstract}
Frank J M van Kuppeveld, associate professor experimental virology, 1,5,6 Arjan S de Jong, molecular medical microbiologist, ${ }^{1,5}$ Kjerstin H Lanke, research technician, ${ }^{1,5,6}$ Gerald W Verhaegh, senior research fellow, 2,6 Willem J G Melchers, molecular medical microbiologist, ${ }^{1,5,6}$ Caroline M A Swanink, medical microbiologist, ${ }^{1}$ Gijs Bleijenberg, professor psychology, ${ }^{4,5,7}$ Mihai G Netea, professor experimental internal medicine, ${ }^{3,5}$ Jochem M D Galama, professor clinical virology, ${ }^{1,5}$ Jos W M van der Meer, professor internal medicine ${ }^{3,5}$
\end{abstract}

\author{
'Department of Medical \\ Microbiology, Radboud University \\ Nijmegen Medical Centre, 6500 \\ HB Nijmegen, Netherlands \\ ${ }^{2}$ Department of Urology, Radboud \\ University Nijmegen Medical \\ Centre \\ ${ }^{3}$ Department of Internal Medicine, \\ Radboud University Nijmegen \\ Medical Centre \\ ${ }^{4}$ Expert Centre for Chronic \\ Fatigue, Radboud University \\ Nijmegen Medical Centre \\ ${ }^{5}$ Nijmegen Institute for Infection, \\ Inflammation, and Immunity, \\ Nijmegen \\ ${ }^{6}$ Nijmegen Centre for Molecular \\ Life Sciences, Nijmegen \\ ${ }^{7}$ Nijmegen Centre for Evidence \\ Based Practice, Nijmegen \\ Correspondence to: F J M van \\ Kuppeveld \\ f.vankuppeveld@ncmls.ru.nl \\ Cite this as: BMJ 2010;340:c1018 \\ doi:10.1136/bmj.c1018
}

\section{ABSTRACT}

Objective The presence of the retrovirus xenotropic murine leukaemia virus-related virus (XMRV) has been reported in peripheral blood mononuclear cells of patients with chronic fatigue syndrome. Considering the potentially great medical and social relevance of such a discovery, we investigated whether this finding could be confirmed in an independent European cohort of patients with chronic fatigue syndrome.

Design Analysis of a well defined cohort of patients and matched neighbourhood controls by polymerase chain reaction.

Setting Certified (ISO 15189) laboratory of clinical virology in a university hospital in the Netherlands.

Population Between December 1991 and April 1992, peripheral blood mononuclear cells were isolated from 76 patients and 69 matched neighbourhood controls. In this study we tested cells from 32 patients and 43 controls from whom original cryopreserved phials were still available.

Main outcome measures Detection of XMRV in peripheral blood mononuclear cells by real time polymerase chain reaction assay targeting the XMRV integrase gene and/or a nested polymerase chain reaction assay targeting the XMRV gag gene.

Results We detected no XMRV sequences in any of the patients or controls in either of the assays, in which relevant positive and negative isolation controls and polymerase chain reaction controls were included. Spiking experiments showed that we were able to detect at least 10 copies of XMRV sequences per $10^{5}$ peripheral blood mononuclear cells by real time as well as by nested polymerase chain reaction, demonstrating high sensitivity of both assays.

Conclusions This study failed to show the presence of XMRV in peripheral blood mononuclear cells of patients with chronic fatigue syndrome from a Dutch cohort. These data cast doubt on the claim that XMRV is associated with chronic fatigue syndrome in the majority of patients.

\section{INTRODUCTION}

Chronic fatigue syndrome, also named myalgic encephalitis, is characterised by disabling physical and mental fatigue, lasting for at least six months, without an apparent physical cause. ${ }^{1-3}$ The hallmark of the illness is debilitating fatigue, but symptoms like myalgia, disrupted sleep, difficulty with concentration, sore throat, and lymphadenopathy may also be present, albeit more variably. More than two thirds of patients are women. Although the cause is unknown and the illness may cover more than one entity, many have suggested that infectious agents have a role. ${ }^{4}$ Indeed, the onset of chronic fatigue syndrome is often preceded by an acute flu-like illness or infectious mononucleosis with seemingly impaired recovery. ${ }^{5}$ A role of chronic infection and changed immunity has been postulated. Most cases of the illness are sporadic, but some clustered cases have been described, particularly suggesting an infectious cause. However, despite extensive studies, no causative infectious agent has been conclusively identified, neither has an immune defect been established to explain the symptoms. ${ }^{26}$

In a recent publication in Science, Lombardi et $\mathrm{al}^{7}$ reported the detection of xenotropic murine leukaemia virus-related virus (XMRV) - a human gamma retrovirus that was first identified in tumour tissue of patients with prostate cancer ${ }^{8}$ - in peripheral blood mononuclear cells of patients with chronic fatigue syndrome. In that study, XMRV was detected by polymerase chain reaction in 67\% of patients (68 of 101 samples) and in $4 \%$ of healthy individuals (eight of 213 samples). Furthermore, antibodies to XMRV were identified in the blood of patients but not in controls. Lombardi et al showed that XMRV was infectious and transmittable from clinical material of patients to $T$ cell cultures and a 
permissive cell line. The genetic sequence of XMRV in patients was nearly identical to that in patients with prostate cancer, indicating that the identified retrovirus is a genuine human virus rather than a mouse leukaemia virus contamination.

This report was considered a major scientific breakthrough and attracted a lot of attention. However, the paper fell short in the description of the patients: what was the nature of the cohort, what was the age and sex distribution, how well were the controls matched? Investigation of an independent cohort is therefore necessary before a causal association between XMRV infection and the development of chronic fatigue syndrome can be ascertained. We investigated the presence of XMRV in a well established Dutch cohort of patients with chronic fatigue syndrome using previously described real time and nested polymerase chain reaction assays on two different target genes. ${ }^{7-9}$

\section{METHODS}

\section{Patient cohort}

All patients and controls examined in this study were part of a Dutch cohort of 298 patients, which has been described in detail. ${ }^{1011}$ All patients of this cohort fulfilled the Oxford criteria and reported severe, unexplained, debilitating fatigue of at least one year in duration. ${ }^{12}$ The median duration of their symptoms was seven years (range 2-45 years). The enrolled patients came to our outpatient clinic twice in a three month period. On the second visit, each patient was accompanied by a neighbourhood control (who was selected by the patient) of the same sex and within two years of the same age. Patients and controls visited our clinic between December 1991 and April 1992. All patients underwent a physical examination and an extensive laboratory work-up and completed a set of questionnaires. ${ }^{10}$ Blood samples were obtained from 76 patients (randomly chosen using a table of random numbers ${ }^{10}$ from the 298 patients described above) and 69 matched neighbourhood controls. Blood samples were sent to the central laboratory of the blood transfusion service in Amsterdam, where peripheral blood mononuclear cells were isolated for a study of lymphocyte subsets and apoptosis. ${ }^{11}$ After isolation, a fraction of the peripheral blood mononuclear cells was directly cryopreserved according to a standard protocol in a computerised device. Cells were aliquoted in phials and stored with $10 \%$ dimethyl sulfoxide at $-196^{\circ} \mathrm{C}$ (liquid nitrogen) in a density of about $10^{7}$ cells per ml. The quality of the storage conditions at the central laboratory of the blood transfusion service has been amply demonstrated by Jansen et al, who showed that peripheral blood mononuclear cells stored for 12 years remained fully viable and immunologically competent. $^{13}$

In this study, we examined peripheral blood mononuclear cells of all patients $(n=32)$ and controls $(n=43)$ from whom original cryopreserved vials were still available. This group included 25 patients and their matched controls, as well as seven patients and 18 controls that were not matched to each other. The male to female ratio of the patient group that was tested in this study was 1:2. Average age of the male patients was 40 . 7 years (range 25-61) and of female patients was 40 . 5 years $(25-67)$.

\section{Nucleic acid isolation and copy DNA synthesis}

Nucleic acid was isolated from $100 \mu \mathrm{l}$ of peripheral blood mononuclear cells (about 0.5 to $2 \times 10^{6}$ cells) using the MagNA-Pure LC and the MagNA-Pure LC Total Nucleic Acid Isolation Kit (Roche Diagnostics, Almere, Netherlands) according to the instructions of the manufacturer and eluted in $50 \mu \mathrm{l}$ of elution buffer. A fixed amount of phocine distemper virus, a paramyxovirus that was used as internal control, was added to the samples before nucleic acid isolation so that we could monitor RNA quality and possible inhibition of amplification of the samples. ${ }^{14}$ RNA in the total nucleic acid isolates was reverse transcribed to copy DNA using the TaqMan Reverse Transcription Reagents kit (Applied Biosystems, Nieuwerkerk aan den IJssel, Netherlands) in a $50 \mu \mathrm{l}$ reaction mix containing $20 \mu \mathrm{l}$ of nucleic acid isolate (concentration 25$150 \mathrm{ng}$ per $\mu \mathrm{l}$ ) and random hexamers as primers, according to the manufacturer's instructions.

\section{Real time polymerase chain reaction assay}

A duplex real time polymerase chain reaction assay was developed, adapted from the XMRV integrase real time polymerase chain reaction assay described by Schlaberg et al, ${ }^{9}$ to detect XMRV and phocine distemper virus simultaneously. The reaction mixture contained $12.5 \mu \mathrm{l}$ of $2 \mathrm{X}$ LightCycler 480 Probes Master (Roche Diagnostics), $1 \mu \mathrm{M}$ of each primer and $400 \mathrm{nM}$ of each probe, and $5 \mu \mathrm{l}$ of copy DNA in a reaction volume of $25 \mu \mathrm{l}$. The XMRV and phocine distemper virus primers were as described. ${ }^{914}$ The XMRV probe was used as a 5 -(6-carboxyfluorescein)-labelled, locked nucleic acid hydrolysis probe and the phocine distemper virus probe was used as a $5^{\prime}$-yakima yellow-labelled, locked nucleic acid hydrolysis probe. All primers and probes used in this study are shown in the table. Cycling conditions were $95^{\circ} \mathrm{C}$ for five minutes, followed by 50 cycles of $95^{\circ} \mathrm{C}$ for 15 seconds and $60^{\circ} \mathrm{C}$ for 45 seconds using the LightCycler 480 instrument (Roche Diagnostics). The result of the sample was considered a valid result only if the crossing point value for the spiked phocine distemper virus was within two cycles of the average of uninhibited samples.

Positive and negative controls for isolation, reverse transcription, and polymerase chain reaction were included in each run. $\beta$-globin real time polymerase chain reaction was performed using primers and hybridisation probes as described. ${ }^{15}$ Mean crossing point value of $\beta$-globin real time polymerase chain reaction assay was 23.84 , standard deviation 0.95 . As a positive control for the polymerase chain reaction assay, we used nucleic acid isolated from $22 \mathrm{Rv} 1$, a prostate carcinoma cell line (American Type Culture Collection number CRL-2505) that was recently shown to contain multiple integrated copies of XMRV and to produce 
high levels of infectious virus. ${ }^{16}$ Total nucleic acid isolation and sample preparation from this cell line was as described above for peripheral blood mononuclear cells.

To determine the sensitivity of the XMRV real time polymerase chain reaction assay, we generated a 192 base pairs XMRV integrase polymerase chain reaction product using primers XMRV-F2 (which is located upstream of XMRV-F1) and XMRV-R3 (which is located downstream of XMRV-R2), and 22Rv1 copy DNA as a template. The polymerase chain reaction product was purified using the Wizard PCR preps DNA purification system (Promega Benelux, Leiden, Netherlands). The concentration was determined using a NanoDrop 1000 (Thermo Scientific/Isogen, De Meern, Netherlands) and the number of copies per $\mu \mathrm{l}$ was calculated. A dilution series was made in which $10^{1}$ to $10^{7}$ copies of the calibrator were added to $10^{6}$ peripheral blood mononuclear cells before nucleic acid isolation. This corresponds to 1 to $10^{6}$ copies per reaction, since a tenth of the isolated nucleic acid was used as input for the polymerase chain reaction, which was performed as described above.

Nested polymerase chain reaction assay

The XMRV gag nested polymerase chain reaction assay was adapted from Urisman et al. ${ }^{8}$ The reaction mixtures contained $25 \mu \mathrm{l}$ of $2 \mathrm{X}$ PCR Master (Roche Diagnostics), and $200 \mathrm{nM}$ of each primer in a reaction volume of $50 \mu \mathrm{l}$. In the first reaction, $5 \mu \mathrm{l}$ of copy DNA was used. Subsequently, $5 \mu \mathrm{l}$ of the first reaction was used as input for the nested reaction. Primers were as

\begin{tabular}{|c|c|c|}
\hline \multicolumn{3}{|c|}{ Sequences of primers and probes used in this study } \\
\hline & Sequence & Reference \\
\hline \multicolumn{3}{|c|}{ XMRV integrase gene } \\
\hline XMRV-F1 & 5'-CGAGAGGCAGCCATGAAGG-3' & 9 \\
\hline XMRV-F2 & $5^{\prime}$-AACCTGATGGCAGATCAAGC-3' & This study \\
\hline XMRV-R1 & 5'-GAGATCTGTTTCGGTGTAATGGAAA-3' & 9 \\
\hline XMRV-R2 & 5'-CCCAGTTCCCGTAGTCTTTGAG-3' & 9 \\
\hline XMRV-R3 & 5'-TTGCCTTGTAGGACCCAAT-3' & This study \\
\hline XMRV-Probe & 5'-AGTTCTAGAAACCTCTACACTC- $3^{\prime}$ & 9 \\
\hline \multicolumn{3}{|c|}{ XMRV gag gene } \\
\hline GAG-O-F & 5'-CGCGTCTGATTTGTTTTGTT-3' & 8 \\
\hline GAG-O-R & 5'-CCGCCTCTTCTTСАTGTTC-3' & 8 \\
\hline GAG-I-F & 5'-TCTCGAGATCATGGGACAGA-3' & 8 \\
\hline GAG-I-R & 5'-AGAGGGTAAGGGCAGGGTAA-3' & 8 \\
\hline GAG-I-R2 & 5'-CAGACTGGTTGGATGCAATG-3' & This study \\
\hline GAG-UNIQ-F & 5'-GACTITTGGAGTGGCTTTGT-3' & 17 \\
\hline \multicolumn{3}{|c|}{ PDV haemagglutinin gene } \\
\hline PDV-F & 5'-GGTGGGTGCCTITACAAGAAC-3' & 14 \\
\hline PDV-R & 5'-ATCTTCTTCCTCAACCTCGTCC-3' & 14 \\
\hline PDV-probe & 5'-ATGCAAGGGCCAATT-3' & 14 \\
\hline \multicolumn{3}{|c|}{ Human $\beta$-globin gene } \\
\hline bGLO-for & 5'-GAGCCATCTATTGCTTACATTTGC-3' & 15 \\
\hline bGLO-rev & 5'-TTGGTCTCCTTAAACCTGTCTTGT-3' & 15 \\
\hline bGLO-FL & 5'-CCAGGGCCTCACCACCAACTTC-3' & 15 \\
\hline bGLO-LC640 & 5'-CCACGTTCACCTTGCCCCACAG-3' & 15 \\
\hline
\end{tabular}

described, except for the reverse primer of the nested reaction (GAG-I-R), which was replaced by GAG-I-R2 to yield a 92 base pairs reaction product (we used primer GAG-I-R2 because it produced less background in the nested reverse transcription polymerase chain reaction). The target sequence of GAG-I-R2 is 100\% conserved among all XMRV isolates published to date (data not shown). Cycling conditions were as previously described. ${ }^{8}$ Polymerase chain reaction products $(20 \mu \mathrm{l})$ were analysed on a $2.5 \%$ agarose gel.

To determine the sensitivity of our XMRV nested polymerase chain reaction assay, a 708 base pairs XMRV gag polymerase chain reaction product was
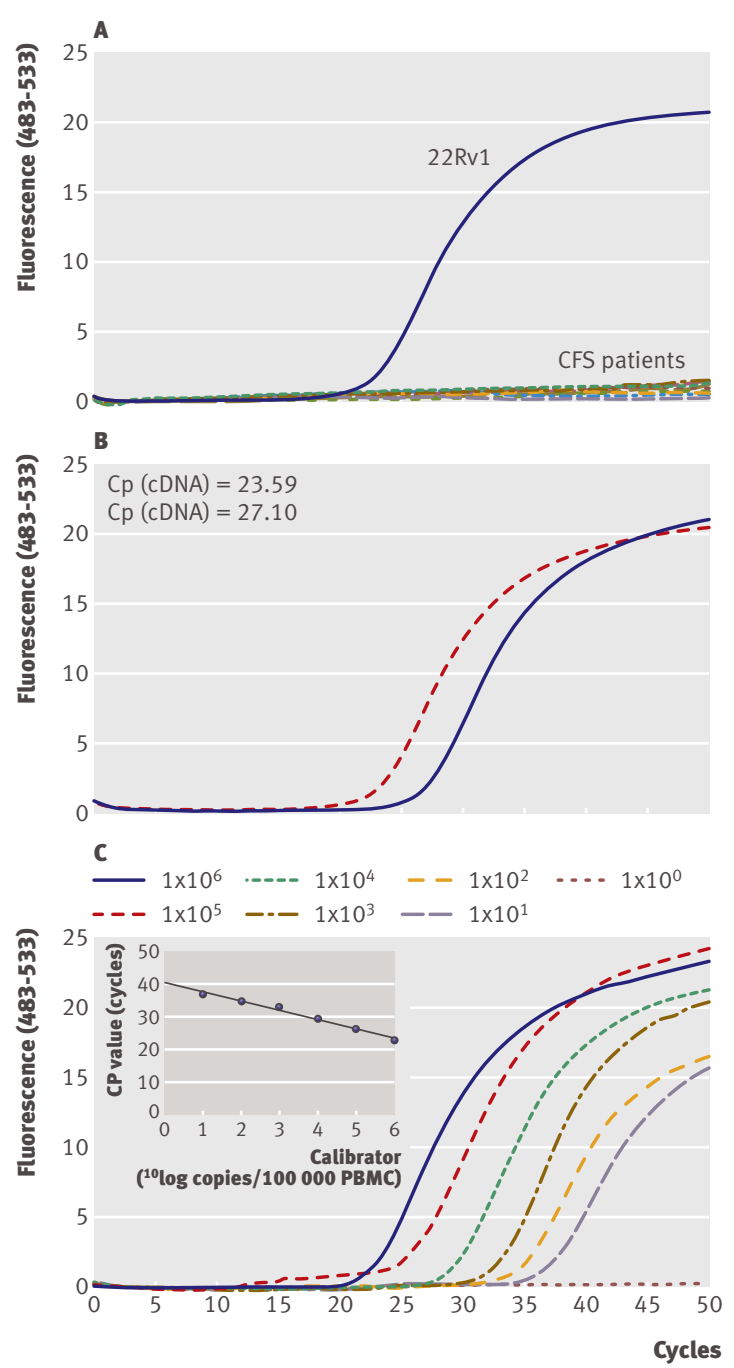

Fig 1| Results of XMRV integrase real time polymerase chain reaction assay. (A) All 32 patients with chronic fatigue syndrome (CFS) compared with positive 22Rv1 control, which yielded a crossing point value of about 23. Results for neighbourhood controls not shown. (B) 22Rv1 total nucleic acid (DNA, solid), reverse transcribed total nucleic acid (cDNA, dashed). The additional reverse transcription step increased the sensitivity of the polymerase chain reaction, decreasing the crossing point $(C p)$ value by 3.5. One of three independent experiments is shown. (C) Sensitivity of the assay. The inlay shows linear relation between number of spiked molecules and crossing point value from $10^{1}$ to $10^{6}$ copies per reaction 
generated using primers GAG-UNIQ-F described by Dong et $\mathrm{al}^{17}$ (which is located upstream of GAG-O-F) and GAG-O-R, and 22Rv1 copy DNA as a template. Purification and determination of the amount of the polymerase chain reaction product were performed as described above for the real time polymerase chain reaction calibrator. A dilution series was made and $10^{1}$ to $10^{7}$ copies of the calibrator were added to $10^{6}$ peripheral blood mononuclear cells prior to nucleic acid isolation. This corresponds to 1 to $10^{6}$ per reaction since a tenth of the isolated nucleic acid was used as input for the nested polymerase chain reaction, which was performed as described above. In the same way, we tested the sensitivity of the nested polymerase chain reaction assay described by Urisman et $\mathrm{al}^{8}$ using primer GAG-I-R instead of GAG-I-R2.

\section{RESULTS}

Total nucleic acid was isolated from peripheral blood mononuclear cells of 32 patients and 43 healthy controls. Nucleic acid was subjected to copy DNA synthesis to increase the sensitivity of our polymerase chain reaction assays. This was done because we observed that the real time polymerase chain reaction assay on nucleic acid isolated from a XMRV positive prostate cancer cell line, 22Rv1, and subjected to copy DNA synthesis - allowing detection of both proviral DNA and viral RNA - was about 10 times more sensitive than without copy DNA synthesis (fig 1B). Nevertheless, all samples from patients with chronic fatigue syndrome and from controls tested negative for both the $\mathrm{XMRV}$ integrase gene (fig 1) and the XMRV gag gene (fig 2).

Our negative XMRV polymerase chain reaction results were unlikely to be due to low amounts of nucleic acid tested or low sensitivity of the assays used. We used 50-300 ng of total nucleic acid from peripheral blood mononuclear cells per polymerase chain reaction, which is similar to the amount used by Lombardi et al. ${ }^{7}$ Moreover, by adding 10-fold serial dilutions of a defined amount of template DNA to peripheral blood mononuclear cells before nucleic acid isolation, we demonstrated that both the real time polymerase chain reaction assay (fig $1 \mathrm{C}$ ) and the nested polymerase chain reaction assay (fig $2 \mathrm{~B}$ ) could detect at least 10 copies of XMRV per $10^{5}$ peripheral blood mononuclear cells, indicating a high sensitivity. A similar sensitivity of the nested polymerase chain reaction assay was observed when we used the same primers as described by Urisman et al (inner reverse primer GAG-I-R instead of the inner reverse primer GAG-I-R2 used in our assay) (data not shown). ${ }^{8}$

Our negative XMRV polymerase chain reaction results are also unlikely to be due to problems with nucleic acid isolation, loss of RNA or DNA integrity, synthesis of copy DNA, or the polymerase chain reaction procedure, since both the phocine distemper virus RNA (an internal control of which a fixed amount was added to each of the samples before nucleic acid isolation) and the $\beta$-globin gene were efficiently amplified in all samples tested (data not shown).
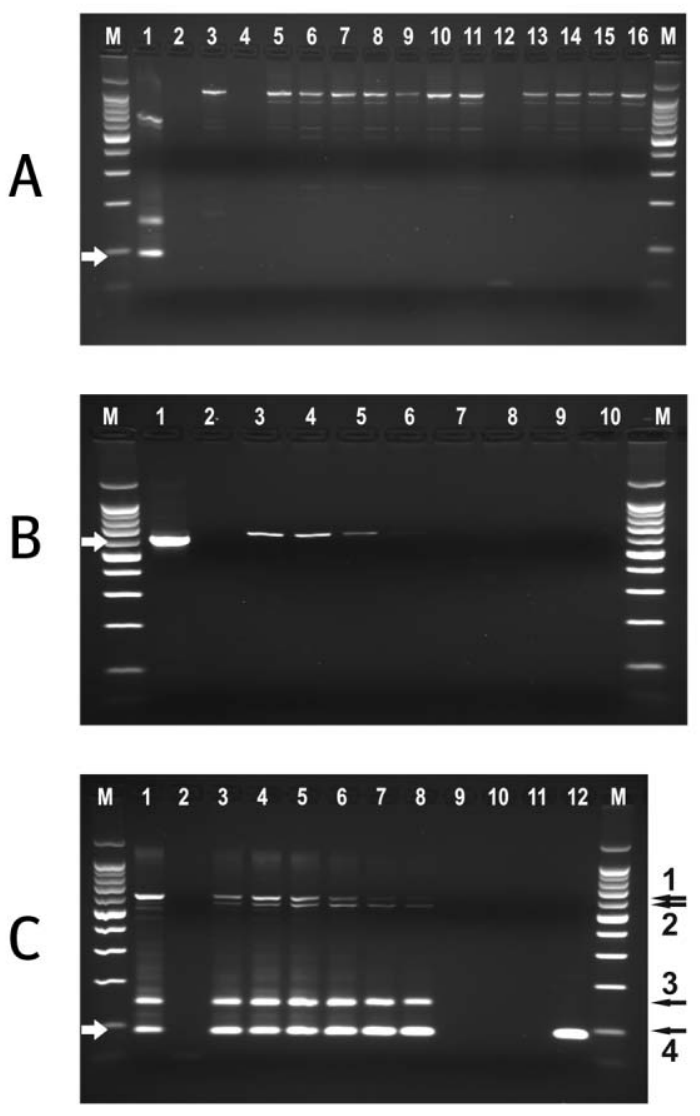

D

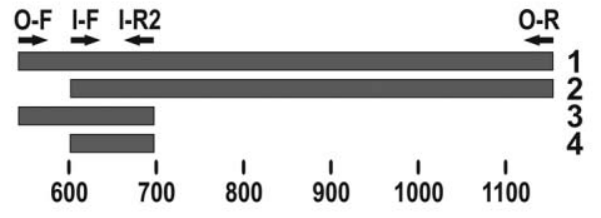

Fig 2 | Results of XMRV gag nested polymerase chain reaction assay. (A) Results for 11 patients with chronic fatigue syndrome and negative controls. Results for neighbourhood controls not shown. (1) The positive 22Rv1 control yielded a product of the expected size of 92 base pairs (arrow); (2) negative polymerase chain reaction control; (3) phocine distemper virus (internal control); (4) negative reverse transcription control; (5)-(11) and (13)-(16) patient samples; (12) negative isolation control; (M) 100 base pairs size marker. (B) Sensitivity of the XMRV gag first polymerase chain reaction. (C) Sensitivity of the nested reaction. White arrows indicate the 613 base pairs (B) and 92 base pairs (C) reaction products. (1) 22Rv1; (2) negative polymerase chain reaction control; (3-9), dilution series of $10^{6}$ to $10^{\circ}$ copies of calibrator per reaction; (10) negative isolation control; (11) negative nested polymerase chain reaction control; (12) positive nested polymerase chain reaction control (22Rv1); (M) 100 base pairs size marker. Black arrows 1-4 in (C) indicate polymerase chain reaction products that are formed in the nested reaction (see D). (D) Positions of the gag primers and the gag polymerase chain reaction products formed in the nested reaction. In addition to the primers of the nested reaction (GAG-I-F, I-F and GAG-I-R2, I-R2), primers from the first reaction (GAG-O-F, O-F and GAG-O-R, O-R) are also present in the nested reaction, yielding reaction products 1-4 that correspond to the black arrows in (C). Numbers represent the positions on the XMRV genome (VP42, accession DQ241302) 


\section{WHAT IS ALREADY KNOWN ON THIS TOPIC}

Chronic fatigue syndrome is a debilitating disease of unknown cause that affects millions of people worldwide

A study from the United States reported the detection of the retrovirus xenotropic murine leukaemia virus-related virus (XMRV) in peripheral blood mononuclear cells in a cohort of patients with chronic fatigue syndrome, suggesting a possible causal relation and a satisfactory explanation for their problems

\section{WHAT THIS PAPER ADDS}

We found no evidence for the occurrence of XMRV in peripheral blood mononuclear cells of patients with chronic fatigue syndrome from a well defined Dutch cohort

These data cast doubt on the claim that XMRV is associated with chronic fatigue syndrome in the majority of patients
As technical aspects do not seem to provide an explanation, the difference might be explained by the two cohorts studied. Our patients met the Oxford criteria for chronic fatigue syndrome, whereas the patients studied by Lombardi et al were reported to fulfil the Centers for Disease Control criteria, ${ }^{7}$ but this is unlikely to explain the absence of XMRV in our patients' samples. Unfortunately, the paper of Lombardi and colleagues lacked a clear description of their patient cohort. Recently, at the Tri-Society Annual Conference 2009 in Lisbon, a presentation reported that the peripheral blood mononuclear cells were derived from patients from the outbreak of chronic fatigue syndrome at Incline village at the northern border of Lake Tahoe, United States (19845). ${ }^{19}$ This outbreak has long been thought to have been caused by a viral infection and has been associated with a number of viruses, most notably Epstein-Barr virus ${ }^{20}$ and human herpes virus $6,{ }^{21}$ but firm evidence for a role of viruses in this particular outbreak has never been provided. It is possible that the study of Lombardi et al has unravelled the viral cause of the chronic fatigue syndrome outbreak, but it seems unlikely that their study demonstrates a viral association for sporadic chronic fatigue syndrome cases, such as those we tested, or represents the majority of patients. Studies of XMRV in sporadic chronic fatigue syndrome cases from the United States would be of great interest.

XMRV was initially identified in tumour tissue of about $10 \%$ of patients with prostate cancer in the United States. ${ }^{8}$ This association was recently confirmed in another independent study from the United States, in which XMRV was detected in $23 \%$ of patients. $^{9}$ Remarkably, in three independent European cohorts of patients with prostate cancer, no XMRV was detected. ${ }^{22-24}$ Whether this discrepancy is due to differences in the geographic distribution of the virus remains to be established.

Recently, a team from the United Kingdom reported the failure to detect XMRV in all 186 tested peripheral blood mononuclear cell samples from a well characterised cohort of British patients with chronic fatigue syndrome. ${ }^{25}$ This team, however, did not use the same primer sets as used by Lombardi et al, leaving open a possible explanation for the difference in results. In our study, we used the same primer sets as used by Lombardi et al. Although our patient group was relatively small and more research is required, our findings - together with those of Erlwein et $\mathrm{al}^{25}$ cast doubt on the claim that XMRV is associated with chronic fatigue syndrome in the majority of patients.

Lombardi et al also detected XMRV in about $4 \%$ of healthy controls. ${ }^{7}$ We failed to detect XMRV in peripheral blood mononuclear cells of healthy controls in our study, but the number of controls tested $(n=43)$ is too low to exclude the occurrence of XMRV in blood in a part of the population. Clearly, more research is needed to establish the distribution of XMRV in healthy controls and, of course, in blood supply products in Europe and in the United States. 


\section{Implications}

In conclusion, we found no evidence for a role of $\mathrm{XMRV}$ in the cause of chronic fatigue syndrome in Dutch patients. Over the past decades we have seen a series of papers prematurely claiming the discovery of the microbial cause of chronic fatigue syndrome. Regrettably, thus far none of these claims has been substantiated.

Contributors: FJMvK, MGN, JMDG, and JWMvdM designed the study and wrote the paper. ASd], KHL, GWV, and WJGM performed experiments and analysed the data. CMAS, GB, JMDG, and JWMvdM established the chronic fatigue syndrome patient cohort. All authors had full access to all of the data (including statistical reports and tables) in the study and take responsibility for the integrity of the data and the accuracy of the data analysis. FJMvK and JWMvdM are guarantors of the paper and accept full responsibility for the work and/or the conduct of the study, had access to the data, and controlled the decision to publish.

Funding: None.

Competing interests: All authors have completed the Unified Competing Interest form at www.icmje.org/coi_disclosure.pdf (available on request from the corresponding author) and declare that none of them (1) has support from companies for the submitted work; (2) has relationships with companies that might have an interest in the submitted work in the previous 3 years; (3) has spouses, partners, or children that have financia relationships that may be relevant to the submitted work, and (4) has non-financial interests that may be relevant to the submitted work. Ethical approval: Ethical aspects of this study were approved by the Commissie Mensgebonden Onderzoek from Radboud University Medical Centre (CMO-1991).

Data sharing: No additional data available.

1 Fukuda K, Straus SE, Hickie I, Sharpe MC, Dobbins JG, Komaroff A. The chronic fatigue syndrome: a comprehensive approach to its definition and study. International Chronic Fatigue Syndrome Study Group. Ann Intern Med 1994;121:953-9.

2 Prins JB, van der Meer JW, Bleijenberg G. Chronic fatigue syndrome. Lancet 2006;367:346-55.

3 Cho HJ, Skowera A, Cleare A, Wessely S. Chronic fatigue syndrome: an update focusing on phenomenology and pathophysiology. Curr Opin Psychiatry 2006:19:67-73.

4 Swartz MN. The chronic fatigue syndrome - one entity or many? N Engl J Med 1988;319:1726-8.

5 White PD, Thomas IM, Kangro HO, Bruce-Jones WDA, Amess I, Crawford DH, et al. Predictions and associations of fatigue syndromes and mood disorders that occur after infectious mononucleosis. Lancet 2001:358:1946-54

6 Lyall M, Peakman M, Wessely S. A systematic review and critical evaluation of the immunology of chronic fatigue syndrome. J Psychosom Res 2003:55:79-90.

7 Lombardi VC, Ruscetti FW, Das Gupta J, Pfost MA, Hagen KS, Peterson DL, et al. Detection of an infectious retrovirus, XMRV, in blood cells of patients with chronic fatigue syndrome. Science 2009;326:585-9.

8 Urisman A, Molinaro RJ, Fisher N, Plummer SJ, Casey G, Klein EA, et al. Identification of a novel Gammaretrovirus in prostate tumors of patients homozygous for R462Q RNASEL variant. PLos Pathog 2006;2:211.

9 Schlaberg R, Choe DJ, Brown KR, Thaker HM, Singh IR. XMRV is present in malignant prostatic epithelium and is associated with prostate cancer, especially with high-grade tumors. Proc Natl Acad Sci USA 2009;106:16351-56

10 Vercoulen JH, Swanink CM, Fennis JF, Galama JM, van der Meer JW, Bleijenberg G. Dimensional assessment of chronic fatigue syndrome. I Psychosom Res 1994;38:383-92.

11 Swanink CM, Vercoulen JH, Galama JM, Roos MT, Meyaard L, van der Ven-Jongekrijg J, et al. Lymphocyte subsets, apoptosis, and cytokines in patients with chronic fatigue syndrome. I Infect Dis 1996;173:460-3.

12 Sharpe MC, Archard LC, Banatvala JE, Borysiewicz, LK, Clare AW, David A, Edwards RH, et al. A report-chronic fatigue syndrome: guidelines for research. J $R$ Soc Med 1991;84:118-21.

13 Jansen CA, de Cuyper IM, Hooibrink B, van der Bij AK, van Baarle D, Miedema F. Prognostic value of the HIV-I gag-specific CD4+ T-cell responses for progression to AIDS analyzed in a prospective cohort study. Blood 2006;107:1427-33.

14 Clancy A, Crowley B, Niesters H, Herra C. The development of a qualitative real time RT-PCR for the detection of hepatitis C virus. Eur Clin Microbiol Infect Dis 2008;27:1177-82.

15 Hesselink AT, van den Brule AJ, Groothuismink ZM, Molano M, Berkhof J, Meijer CJ, et al. Comparison of three different PCR methods for quantifying human papillomavirus type 16 DNA in cervical scrape specimens. J Clin Microbiol 2005;43:4868-71.

16 Knouf EC, Metzger MJ, Mitchell PS, Arroyo JD, Chevillet JR, Tewari M, et al. Multiple integrated copies and high-level production of the human retrovirus XMRV (xenotropic murine leukemia virus-related virus) from 22 Rv1 prostate carcinoma cells. J Virol 2009;83:7353-6.

17 Dong B, Kim S, Hong S, Das Gupta J, Malathi K, Klein E, et al. An infectious retrovirus susceptible to an IFN antiviral pathway from human prostate tumors. Proc Natl Acad Sci USA 2007;104:1655-60.

18 Eypasch E, Lefering R, Kum CK, Troidl H. Probability of adverse events that have not yet occurred: a statistical reminder. $B M$ J 1995;311:619-20.

19 Mikovits J. Detection and immune correlates of an infectious retrovirus, XMRV, in blood cells of patients with chronic fatigue syndrome. Lisbon: Tri-Society Annual Conference, 18-21 October 2009. Cytokine 2009;48:10.

20 Holmes GP, Kaplan JE, Stewart JA, Hunt B, Pinsky PF, Schonberger LB A cluster of patients with a chronic mononucleosis-like syndrome. Is Epstein-Barr virus the cause? JAMA 1987;257:2297-302.

21 Daugherty SA, Henry BE, Peterson DL, Swarts RL, Bastien S, Thomas RS. Chronic fatigue syndrome in northern Nevada. Rev Infect Dis 1991;13(Suppl 1):s39-44

22 Fisher N, Hellwinkel O, Schulz C, Chun FK, Huland H, Aepfelbacher M, Schlomm T. Prevalence of human gammaretrovirus XMRV in sporadic prostate cancer. J Clin Virol 2008;43:277-83.

23 Hohn O, Krause H, Barbarotto P, Niederstadt L, Beimforde N, Denner J, Miller K, et al. Lack of evidence for xenotropic murine leucemia virus-related virus (XMRV) in German prostate cancer patients. Retrovirology 2009;6:92.

24 D’Arcy F, Foley R, Perry A, Marignol L, Lawler M, Gaffney E, et al. No evidence of XMRV in Irish prostate cancer patients with the R462Q mutation. Eur Urol 2008; Suppl 7:271.

25 Erlwein O, Kaye S, McClure M, Weber I, Wills G, Collier D, et al. Failure to detect the novel retrovirus XMRV in chronic fatigue syndrome. PLOS ONE 2010;5:e8519.

Accepted: 16 February 2010 